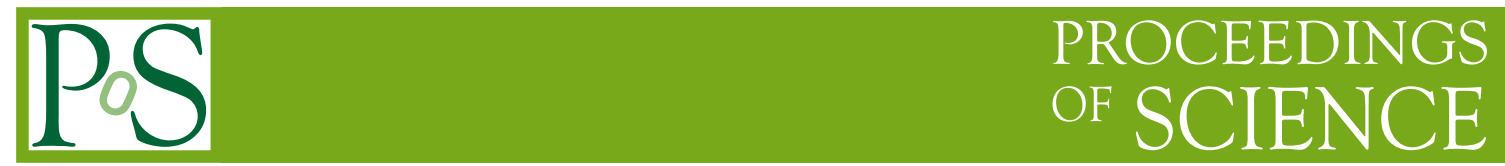

\title{
Multiparticle Cuts of Scattering Amplitudes
}

\author{
Pierpaolo Mastrolia* \\ CERN, CH-1211 Geneva, Switzerland \\ Institute for Theoretical Physics, University of Zürich, CH-8057 Zürich, Switzerland \\ E-mail: Pierpaolo.Mastrolia@cern.ch
}

Generalised unitarity is a very effective tool for computing one-loop amplitudes analitically. I recall the techniques of the quadruple-cuts and the of the double-cuts with the spinor integration of the phase-space, mainly focusing on its extension to compute triple-cuts.

8th International Symposium on Radiative Corrections (RADCOR)

October 1-5 2007

Florence, Italy

\footnotetext{
* Speaker.
} 


\section{Generalised Unitarity}

The recent boost in the development of thecniques for evaluating scattering amplitudes is certainly due to the better understanding of their analytic properties. By relaxing the condition of working with real Minkowski kinematics, and using, instead, complex momenta for either external or propagating particles, off-shell degrees of freedom are reparametrized within complex, yet onshell quantities. On-shell methods make use of complex kinematics for exploiting the singularities of on-shell amplitudes and the factorization information to reconstruct amplitudes iteratively from their poles at the tree-level [1], and from their branch-cuts at higher order [2] - since the singularities of scattering amplitude are determined by lower-point amplitudes in the case of poles, and by lowerloop ones in the case of cuts $[3,4,5]$. On-shell methods were originally used in [6] and in the more recent systematized implementations for the completion of all six-gluon helicity amplitudes (see [8] for a complete list of refs.) and the calculation of the six-photon amplitudes [14, 15].

In this talk I discuss a possible implementation of generalised unitarity as on-shell method for computing one-loop amplitudes analitically. I recall the techniques of the quadruple-cuts and the of the double-cuts with spinor integration, mainly focusing on its extension to compute triple-cuts.

At one loop, generalized unitarity corresponds to requiring more than two internal particles to be on-shell, as realized, in general, only with complex momenta. The application of unitarity as an on-shell method of calculation [7], is based on the principles that: $i$ ) by means of PassarinoVeltman reduction, any one-loop amplitude is expected to be expressed as a linear combination of scalar (i.e. with trivial numerator) master integrals (MI); ii) any MI is identified by the number of its external legs, and characterized by its own set of (leading and subleading) singularities; iii) products of on-shell tree-level amplitudes produce one-loop functions with the proper branch cuts. Provided the knowledge of the MI (that can be apriori evaluated), to compute any amplitude it is sufficient the evaluation of the coefficients of its decomposition in terms of MI. The principle of a unitarity-based method is the extraction of the rational coefficients from the decomposition, by matching the multiparticle cuts of an amplitude onto the corresponding cuts of the MI.

\section{Multiparticle-Cuts in 4- and $D$-dimensions}

Any one-loop amplitude in four dimension can be expressed in terms of poly-logarithms and rational funtions associated to a combination of 2-, 3-, and 4-point functions (the occurrence of 0point functions is not discussed here), see Fig.1. The use of 4-dimensional states and momenta in the cuts enable the construction of the poly-logarithmic terms (plus trascendental constants) of the amplitudes, which are fixed by their branch-cuts, and are called cut-constructible; but generically drops the rational terms that have to be recovered independently.

Recent improvements to the unitarity method use complex momenta within generalized unitarity. The most effective application of such an idea is represented by the quadruple-cut, shown in Fig.2 (line-one). Quadruple-cuts allow for a simple and purely algebraic determination of the coefficients of box-functions, because the four cut-conditions freeze completely the 4-dimensional loop integration. Accordingly, every box-coefficient is simply determined by the product of the four tree-level amplitude sitting at each corner, read at the values of the loop momentum which fulfill the equations imposed by the vanishing of the cut denominators [9]. 


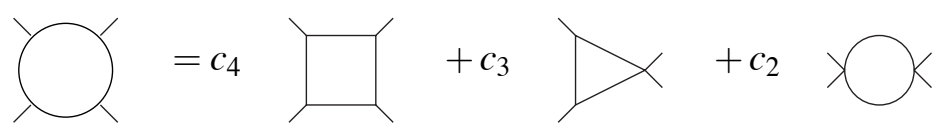

Figure 1: Decomposition of any one-loop amplitude in terms of basic scalar integrals.

Using triple- and double-cuts, respectively shown in Fig.2 (line-two) and (line-three), has led to very efficient techniques for extracting triangle and bubble integral coefficients analytically [10, $11,12,13]$. In fact, while in the case of the quadruple-cut the conditions imposed by the vanishing of four denominators bind completely the loop momentum, in case where less than four denominators are cut, the loop momentum can still run, and the explicit integration over the phase-space is required. In particular in $[10,11]$, the double- and triple-cut phase-space integration has been reduced to the extraction of residues in spinor variables and residues in Feynman-parameters.

The 4-dimensional version of the unitarity method leaves, as said, the pure rational term in the amplitudes undetermined. The so called unitarity bootstrapp method [16] can reconstruct the rational term recursively, due to the interplay of 4-dimensional unitarity and on-shell recurrence relations. Alternatiely, full one-loop amplitudes can be constructed from unitarity cuts in $D=4-2 \varepsilon$ dimensions [17], since in the $D$-dimensional unitarity method, what is intended as "rational" (after expanding around $\varepsilon=0$ ), appears as $\varepsilon$-dependent terms in the coefficients of the MI (before expanding around $\varepsilon=0$ ). A systematic $D$-dimensional unitarity double-cut method was proposed in [18], reducing one-loop amplitudes to MI for arbitrary values of the dimension parameter. Accordingly, the double-cut phase-space in $D$ dimensions is written as a convolution of a 4-dimensional double-cut with an additional mass present in all propagators, and an integration over such mass parameter [20]. Only the 4-dimensional integration is explicitly required, and it can be performed by 4-dimensional unitarity techniques [10]. The remaining integral over the extra parameter, which gives rise to the $\varepsilon$-dependence of the cut-amplitude, is mapped to phase-space integrals in shifted dimensions. It is important to remark that because of the splitting of the loop variable in a 4-dimensional component and a $(-2 \varepsilon)$-complement, the singularities structure (poles and branch-cuts) of a loop integral is exposed after the 4-dimensional integration.

Given the decomposition of any amplitude in terms of MI, the coefficient of any $n$-point MI can be recovered from the $n$-particle cut. Obviously, any $n$-particle cut may detect as well cuts of higher-point MI, which will anyhow appear with different analytic structures for they come from the Landau poles specific to each of the basic scalar integrals. The coefficient of a given $n$-point MI appears in the non-logarithmic term of the corresponding $n$-particle cut, being either a rational or an irrational function. The logarithmic term of a $n$-particle cut can be associated to a function with number of points larger than $n$ : usually logarithms are generated by integrating over variables which are not frozen by the cut-conditions. The non-logarithmic term of a $n$-particle cut can as well contain a $n+1$-point MI: such a situation does happen when the $n+1$-point MI degenerates, namely has the same number of independent scales of a $n$-point MI.

\subsection{Triple-Cut}

In this talk, I show a new way for computing triple-cuts of one-loop amplitudes. It enables the direct extraction of triangle- and higher-point-function coefficients from any one-loop amplitude in arbitrary dimensions. It extends the benefits of the double-cut integration of $[10,18]$, through 

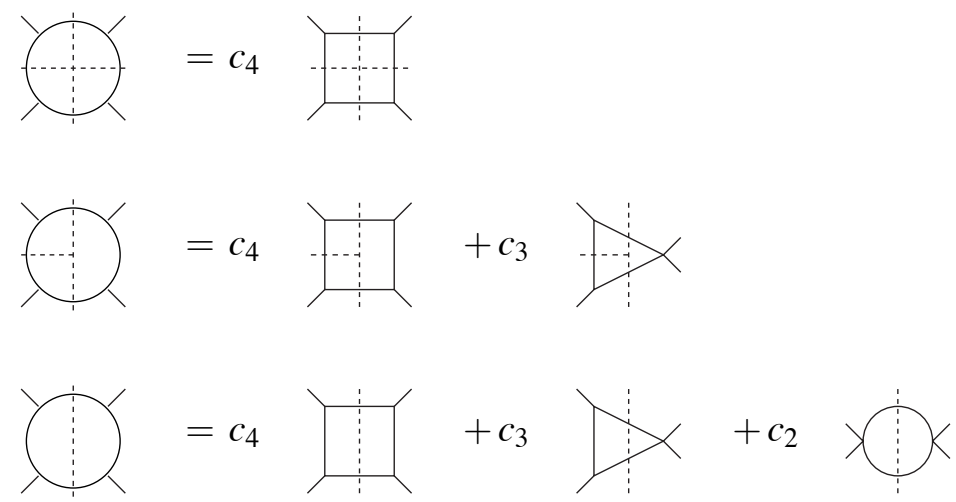

Figure 2: Multiple-cuts of a generic one-loop amplitude expressed in terms of the corresponding cuts of the scalar master integrals: quadruple-cut (line-one), triple-cut (line-two), double-cut (line-three).

the idea of the inverse Cutkosky rule [19] for replacing one of the three on-shell $\delta$-function by the difference of two propagators,

$$
\delta\left(p^{2}-\mu^{2}\right)=\frac{1}{(2 \pi i)}\left\{\frac{1}{p^{2}-\mu^{2}+i 0}-\frac{1}{p^{2}-\mu^{2}-i 0}\right\}
$$

As a consequence, the triple-cut is treated as a difference of two double-cuts with the same particle contents, and a same propagator carrying respectively causal and anti-causal prescription in each of the two cuts, as shown in Fig.3. The triple-cut phase-space in $D$ dimensions is written as a

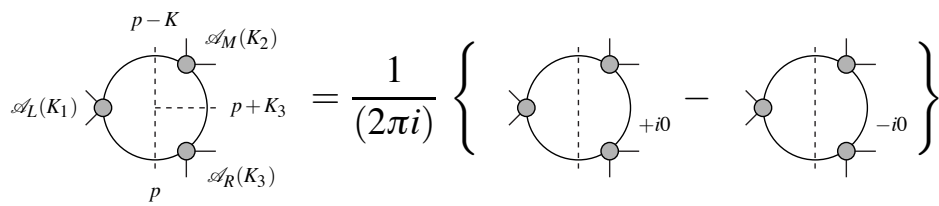

Figure 3: Triple-cut in terms of two double-cuts, respectively with a causal propagator and an anti-causal propagator: $\mathscr{A}_{L}, \mathscr{A}_{M}$, and $\mathscr{A}_{R}$ are tree level amplitudes, respectively depending on the external momenta $K_{1}, K_{2}, K_{3}$.

convolution of a 4-dimensional triple-cut with an additional mass present in all propagators, and an integration over such mass parameter, which plays the role of a $(-2 \varepsilon)$-dimensional scale. As for the double-cut [18], to perform the 4-dimensional integration, one combines the method of spinor integration via the holomorphic anomaly of massive phase-space integrals with an integration over the Feynman parameter. But, in the case of the triple-cut, after Feynman parametrisation and after reading the residues in spinor variables, by combining back the two double-cuts (carrying opposite $i 0$-prescription), the parametric integration is reduced to the extraction of residues in the Feynman parameter, due to relations like

$$
\frac{1}{\left(a x^{2}+b x+c\right)+i 0}-\frac{1}{\left(a x^{2}+b x+c\right)-i 0}=\frac{2 \pi i}{a\left|x_{1}-x_{2}\right|}\left(\delta\left(x-x_{1}\right)+\delta\left(x-x_{2}\right)\right),
$$

where $x_{1,2}$ are the zeroes of the corresponding quadratic denominator. These roots do carry the analytic information that characterizes each MI by determining its own generalised cuts [21]. The final integration over the dimensional scale parameter is mapped, as for the double-cut, directly to triple-cut of master integrals with shifted dimensions. 

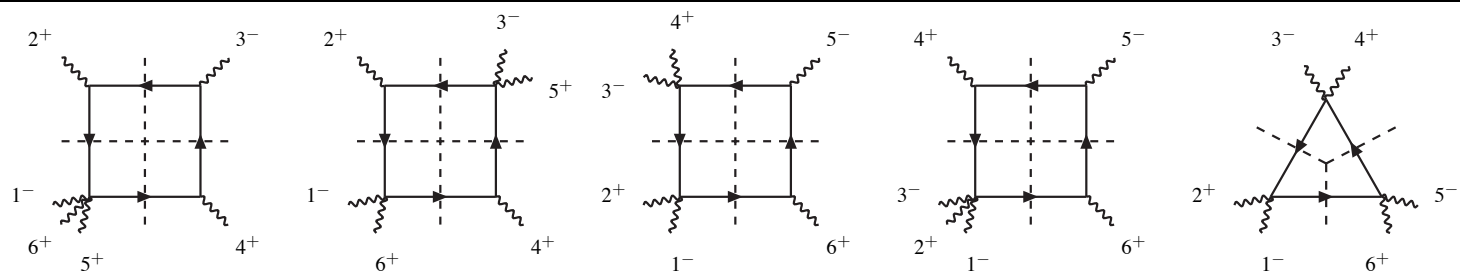

Figure 4: Independent multiple-cuts of the six-photon amplitudes.

\section{Conclusions}

The multiple-cut techniques outlined above have been applied already in several contexts. In particular, the double-cuts based on spinor-integration [10] had been succesfully used to compute analytically the less trivial contributions to the cut-constructible part of the six-gluon amplitude. Recently, quadruple-cuts [9] and triple-cuts [11] were employed for the calculation of the complete six-photon amplitudes [15], whose cut-constructibility, namely the absence of rational ambiguities, was shown in [14]. The absence of rational term in double-cuts indicated the vanishing of 2-points coefficients, therefore the six-photon amplitude could be expressed in terms of box- and trianglefunction associated to the six cut-diagrams shown in Fig.4 (plus permutations). Our analytic results, in agreement with the numerical counterpart [22], was also confirmed by [12]. Double-cuts [10] have been used for the computation of the one-loop MHV amplitude involving a Higgs and an arbitrary number of gluons (two carrying negative helicity, and the rest, positive one) in the heavy-top limit [23]. On-shell methods for tree- and loop-amplitudes, within the spinor-helicity formlism, have been implemented in the Mathematica package S@M [24], which has been used in [15, 23]. Unitarity based methods for one-loop amplitudes have been under an intense development. By now, we dispose of further refined techniques for evaluating the coefficients of the decomposition in terms of MI for generic amplitudes, which minimize the computational load to purely algebraic operations $[25,12]$. They have the potentiality for rendering the evaluation of high-multiplicity one-loop scattering amplitudes completely automatizable, and their numerical implementation has been receiving much attention [26].

In this talk I have discussed one possible analytic implementation of the multiple-cuts, based on the combination of spinor-integration and Feynman parametric integration, in particular focusing on the triple-cut. As it turned out, in the evaluation of any triple-cut, while the spinor integration is carried on by Cauchy's residues theorem, the parametric integration is trivialised by the extra cut-condition. This last feature suggests the applicability of cut-constraints directly in the representation of an amplitude as multiple integral in Feynman parameters, which can be as well applied beyond one-loop.

\section{References}

[1] R. Britto, F. Cachazo and B. Feng, Nucl. Phys. B 715, 499 (2005) [hep-th/0412308]. R. Britto, F. Cachazo, B. Feng and E. Witten, Phys. Rev. Lett. 94, 181602 (2005) [hep-th/0501052].

[2] Z. Bern, L. J. Dixon and D. A. Kosower, Annals Phys. 322, 1587 (2007) [arXiv:0704.2798 [hep-ph]].

[3] S. J. Parke and T. R. Taylor, Phys. Rev. Lett. 56, 2459 (1986); M. L. Mangano and S. J. Parke, Phys. Rept. 200, 301 (1991).

\footnotetext{
${ }^{1}$ ee also the contributions of Darren Forde, Zoltan Kunszt, and Costas Papadopoulos in these Proceedings
} 
[4] Z. Bern and G. Chalmers, Nucl. Phys. B 447, 465 (1995) [hep-ph/9503236].

[5] Z. Bern, L. J. Dixon and D. A. Kosower, Ann. Rev. Nucl. Part. Sci. 46, 109 (1996) [hep-ph/9602280].

[6] Z. Bern, L. J. Dixon and D. A. Kosower, Nucl. Phys. B 513, 3 (1998) [hep-ph/9708239].

[7] Z. Bern, L. J. Dixon, D. C. Dunbar and D. A. Kosower, Nucl. Phys. B 425, 217 (1994) [hep-ph/9403226].

[8] P. Mastrolia, arXiv:hep-ph/0610190.

[9] R. Britto, F. Cachazo and B. Feng, Nucl. Phys. B 725, 275 (2005) [hep-th/0412103].

[10] R. Britto, E. Buchbinder, F. Cachazo and B. Feng, Phys. Rev. D 72, 065012 (2005) [hep-ph/0503132]. R. Britto, B. Feng and P. Mastrolia, Phys. Rev. D 73, 105004 (2006) [hep-ph/0602178].

[11] P. Mastrolia, Phys. Lett. B 644, 272 (2007) [hep-th/0611091].

[12] D. Forde, Phys. Rev. D 75, 125019 (2007) [arXiv:0704.1835 [hep-ph]].

[13] N. E. J. Bjerrum-Bohr, D. C. Dunbar and W. B. Perkins, arXiv:0709.2086 [hep-ph].

[14] T. Binoth, J. P. Guillet and G. Heinrich, JHEP 0702, 013 (2007) [hep-ph/0609054].

[15] T. Binoth, T. Gehrmann, G. Heinrich and P. Mastrolia, hep-ph/0703311.

[16] Z. Bern, L. J. Dixon and D. A. Kosower, Phys. Rev. D 73, 065013 (2006) [hep-ph/0507005]. C. F. Berger, Z. Bern, L. J. Dixon, D. Forde and D. A. Kosower, Phys. Rev. D 74, 036009 (2006) [hep-ph/0604195];. Phys. Rev. D 75, 016006 (2007) [hep-ph/0607014].

[17] W. L. van Neerven, Nucl. Phys. B 268, 453 (1986). Z. Bern and A. G. Morgan, Nucl. Phys. B 467, 479 (1996) [hep-ph/9511336]; Z. Bern, L. J. Dixon, D. C. Dunbar and D. A. Kosower, Phys. Lett. B 394, 105 (1997) [hep-th/9611127]; A. Brandhuber, S. McNamara, B. J. Spence and G. Travaglini, JHEP 0510, 011 (2005) [hep-th/0506068].

[18] C. Anastasiou, R. Britto, B. Feng, Z. Kunszt and P. Mastrolia, Phys. Lett. B 645, 213 (2007) [hep-ph/0609191]; JHEP 0703, 111 (2007) [hep-ph/0612277]; R. Britto and B. Feng, hep-ph/0612089.

[19] R. E. Cutkosky, J. Math. Phys. 1, 429 (1960); C. Anastasiou and K. Melnikov, Nucl. Phys. B 646, 220 (2002).

[20] G. Mahlon, [hep-ph/9311213].

[21] G. 't Hooft, and M. Veltman, Nucl. Phys. B 153 (1979) 365.

[22] Z. Nagy and D. E. Soper, Phys. Rev. D 74, 093006 (2006) [hep-ph/0610028]. G. Ossola, C. G. Papadopoulos and R. Pittau, 0704.1271 [hep-ph].

[23] E. W. N. Glover, P. Mastrolia, and C. Williams, in preparation.

[24] D. Maitre and P. Mastrolia, arXiv:0710.5559 [hep-ph].

[25] G. Ossola, C. G. Papadopoulos and R. Pittau, Nucl. Phys. B 763, 147 (2007) [hep-ph/0609007];

R. Britto and B. Feng, arXiv:0711.4284 [hep-ph]; W. B. Kilgore, arXiv:0711.5015 [hep-ph];

R. K. Ellis, W. T. Giele and Z. Kunszt, arXiv:0708.2398 [hep-ph].

[26] G. Ossola, C. G. Papadopoulos and R. Pittau, arXiv:0711.3596 [hep-ph]. 